\title{
Evaluation of Tobacco Use and Her-2 Receptor Expression in Breast Cancer in an Ethnically Diverse Inner-City Population
}

\author{
Damien M. Hansra, Judith Hurley \\ Sylvester Comprehensive Cancer Center at the University of Miami, Miami, USA \\ Email: dmhansra@med.miami.edu
}

Received 8 March 2014; revised 8 April 2014; accepted 15 April 2014

Copyright (C) 2014 by authors and Scientific Research Publishing Inc. This work is licensed under the Creative Commons Attribution International License (CC BY). http://creativecommons.org/licenses/by/4.0/

(c) (i) Open Access

\begin{abstract}
Background: Tobacco is linked to most cancers however despite overwhelming biological plausibility and decades of epidemiological studies, no association has been established between tobacco and breast cancer. Although estrogen receptor status has been looked at as a variable there has been no evaluation of the role of Her-2 and smoking in breast cancer. Methods: Review of records from patients treated at the University of Miami/Jackson Memorial Hospital from 1998-2012. The incidence of smoking and Her-2 expression in1255 women was evaluated. Data was analyzed by age, race, ethnic group, menopausal status, tumor stage, and ER/PR/Her-2 receptor status. Results: 1255 charts were analyzed with 1094 having full information. Overall rate of Her-2 expression $18.1 \%$. The rate of Her-2 expression was $21.4 \%$ in smokers and $17.0 \%$ in non-smokers $(p=$ 0.10). The rate of Her-2 expression was $10.8 \%$ in Caucasian smokers and $9.8 \%$ in Caucasian nonsmokers ( $p=0.88) ; 24.5 \%$ in smokers of African descent and $17.3 \%$ in non-smokers of African descent ( $p=0.24) ; 22.9 \%$ in Latino smokers and $17.4 \%$ in Latino non-smokers $(p=0.10)$. The rate of Her-2/ER expression was $9.4 \%$ in smokers and $7.9 \%$ in non-smokers $(p=0.42) ; 5.4 \%$ in Caucasian smokers and $4.9 \%$ in Caucasian non-smokers $(p=0.916) ; 12.2 \%$ in smokers of African descent and 5.9\% in non-smokers of African descent ( $p=0.11$ ); $9.5 \%$ in Latin smokers and $8.8 \%$ in Latin non-smokers $(p=0.77)$. Conclusions: We found non-statistically significant positive associations in all analyses between Her-2 expression with or without ER expression and tobacco exposure when analyzed by ethnicity.
\end{abstract}

\section{Keywords}

HER-2, Breast Cancer, Tobacco 


\section{Introduction}

Tobacco still remains the leading cause of preventable death in the US [1] and causes many illnesses, most notably, cancer. Tobacco has been linked to many cancer types and a causative relationship has been established for some malignancies, such as lung cancer [1]. Epidemiological studies are mixed results regarding the effect of tobacco in breast cancer risk [2] [3]. These studies included only estrogen (ER) and progesterone (PR) receptor analysis and the effect of Human Epidermal growth factor Receptor 2 (HER-2) may have a significant association with tobacco and breast cancer risk. We are the first study to examine HER2, ER, PR, tobacco use and breast cancer risk.

\section{Methods}

After Institutional review board approval granted, a retrospective chart analysis was performed involving 1255 patients. Medical records were combined electronic and paper charts from patients treated at the University of Miami Breast Clinic/Jackson Memorial Hospital, Miami, Florida. Two primary investigators performed chart review and data collection. Variables collected included age at diagnosis, stage at diagnosis, race, receptor status, smoking status and number of tobacco pack years. All patients enrolled in study had both paper and electronic charts and both were scanned in entirety for information from original date of consultation for breast cancer to most recent medical records in order to ensure highest accuracy of information extracted.

Inclusion criteria were adult female with breast cancer diagnosed from 1998-2009 who had available documented HER2 expression status, ER positivity and detailed smoking exposure history. To be considered a smoker, the patients had to be current or former cigarette smokers with exposure duration of one year or more. Patients with incomplete, inadequate or ambiguous smoking exposure were excluded, such as social history documented as "negative times 3". Likewise patients who did not have documented HER2 status or who had BRCA mutation were excluded from final analysis. Male patients with breast cancer were excluded as well.

Primary outcome was association between HER2 overexpression and tobacco exposure. Secondary outcome was the association of ER/PR positivity and breast cancer stage with tobacco exposure. Furthermore, primary and secondary outcomes were stratified by race and menopausal status given potential biological differences in these groups. Age 51 was chosen based on United States national average of menopause and not by chart documentation of menopausal status.

Statistical analysis was performed using SPSS version 13.0. Association between HER2 overexpression, ER and PR with tobacco exposure was evaluated by chi-square using a dichotomized approach to these variables which was then expressed as odd-ratios with 95\% confidence intervals. When patients did have tobacco exposure, the intensity of exposure measured by the pack-years mean between dichotomized outcomes was evaluated by t-test. Subgroup analysis was performed by race, ethnicity and menopausal status. We also analyzed tobacco exposure by race and ethnicity.

\section{Results}

A total of 1255 patient's medical records were reviewed. Lack of records completeness resulted in exclusion of 161 patients. Final analysis was performed in a cohort of 1094 (Table 1). Mean age was 56 with range of 22 to 94 years old. Of the 1094 patients 725 (66.3\%) where Latin, 78 (7.1\%) where Caucasian, 286 (26.1\%) were African American, and 5 (0.5\%) were Asian. Of the 1095 patients 198 (18.1\%) overexpressed HER2, 790 (72.2\%) expressed ER, 90 (8.2\%) expressed both ER and HER2, 498 (45.5\%) expressed PR, and 171 (15.6\%) were triple negative. Of the 1094 patients 828 (75.7\%) were never exposed to tobacco and 266 (24.3\%) patients were exposed to tobacco. Of the 266 exposed patients 105 (9.6\%) were actively smoking at time of diagnosis and the remaining 161 (14.7\%) of patients had reportedly quit at time of diagnosis. Duration of quit status was not documented. Smoking rates were significantly higher in Caucasian versus African American women (47.4\% vs. $17.1 \%, \mathrm{P}<0.01)$ and in Latin versus African American women (24.7\% vs. $17.1 \%, \mathrm{P}<0.01)$. Staging demographics of patients upon diagnosis were as follows: stage 1; 257 (23.5\%), stage 2; 372 (34\%), stage 3; 355 (32.4\%), stage 4; 110 (10.1\%).

HER2 receptor analysis: Odds Ratio of having HER2 over expression in patients who smoke vs. nonsmokers is 1.33 ( $p=0.105$; 95\% CI 0.94 - 1.87). Odds ratio of having HER2 over expression in Latin patient who smoke vs. non-smokers is 1.40 ( $\mathrm{p}=0.102$; 95\% CI 0.93 - 2.13). Odds Ratio of having HER2 over expres- 
Table 1. Patient characteristics.

\begin{tabular}{|c|c|c|}
\hline Characteristic & $\mathrm{n}$ & $\%$ \\
\hline Mean age, years & 56 (range 22 - 94) & \\
\hline \multicolumn{3}{|l|}{ Race } \\
\hline Latin & 725 & 66.3 \\
\hline Caucasian & 78 & 7.1 \\
\hline African America & 286 & 26.1 \\
\hline Asian & 5 & 0.5 \\
\hline \multicolumn{3}{|l|}{ Receptor status } \\
\hline ER Positive & 790 & 72.2 \\
\hline PR Positive (missing) & 498(199) & $45.5(18.2)$ \\
\hline HER2 Over-expression & 198 & 18.1 \\
\hline ER/HER2 Over-expression & 90 & 8.2 \\
\hline Triple negative & 171 & 15.6 \\
\hline \multicolumn{3}{|l|}{ Tobacco Exposure } \\
\hline Exposed & 266 & 24.3 \\
\hline Active & 105 & 9.6 \\
\hline Quit & 161 & 14.7 \\
\hline \multicolumn{3}{|l|}{ Stage } \\
\hline Stage I & 257 & 23.5 \\
\hline Stage II & 372 & 34.0 \\
\hline Stage III & 355 & 32.4 \\
\hline Stage IV & 110 & 10.1 \\
\hline
\end{tabular}

sion in Caucasian patients who smoke vs. non smoker is 1.12 ( $\mathrm{p}=0.878$; 95\% CI 0.26 - 4.84). Odds Ratio of having HER2 over expression in African American patients who smoke vs. non- smokers is 1.55 ( $p=0.238$; $95 \%$ CI 0.74 - 3.23). Odds ratio of having HER2 over expression in premenopausal women who smoke vs. nonsmokers is 1.71 ( $\mathrm{p}=0.096$; 95\% CI 0.91 - 3.22); and 1.20 ( $\mathrm{p}=0.379$ 95\% CI 0.80 - 1.82) in post menopausal women

Estrogen receptor analysis: Odds Ratio of having ER over expression in all patients who smoke vs. nonsmokers is 0.95 ( $p=0.74 ; 95 \%$ CI 0.70 - 1.29). Odds Ratio of having ER over expression in all Latin patients who smoke vs. non-smokers is 0.83 ( $\mathrm{p}=0.366$; 95\% CI 0.57 - 1.23). Odds Ratio of having ER over expression in all Caucasian patients who smoke vs. non- smokers is 0.74 ( $p=0.611$; 95\% CI $0.24-2.3$ ). Odds Ratio of having ER over expression in African American patients who smoke vs. non- smokers is 0.99 ( $p=0.997 ; 95 \%$ CI 0.53 - 1.89). Odds Ratio of having ER over expression in premenopausal women who smoke vs. nonsmokers is 1.12 ( $\mathrm{p}=0.700 ; 95 \%$ CI 0.62 - 2.00). Odds Ratio of having ER over expression in postmenopausal women who smoke vs. non- smokers is 0.89 ( $p=0.519$; 95\% CI $0.62-1.28)$.

Estrogen and HER2 receptor analysis: Odds Ratio of having ER/HER2 over expression in all patients who smoke vs. non- smokers is 1.22 ( $\mathrm{p}=0.424$; 95\% CI 0.75 - 1.97). Odds Ratio of having ER/HER2 over expression in all Latin patients who smoke vs. non-smokers is 1.09 ( $p=0.774$; 95\% CI 0.61 - 1.94). Odds Ratio of having ER/HER2 over expression in all Caucasian patients who smoke vs. non- smokers is 1.12 (p = 0.916; $95 \%$ CI 0.15 - 8.34). Odds Ratio of having ER/HER2 over expression in African American patients who smoke vs. non- smokers is 2.22 ( $p=0.113$; 95\% CI 0.81 - 6.10). Odds Ratio of having ER/HER2 over expression in pre- 
menopausal women who smoke vs. non- smokers is 1.56 ( $\mathrm{p}=0.329$; 95\% CI 0.64 - 3.79). Odds Ratio of having ER/HER2 over expression in postmenopausal women who smoke vs. non- smokers is 1.11 ( $\mathrm{p}=0.735$; $95 \% \mathrm{CI}$ 0.62 - 1.97).

Triple negative and progesterone receptor analysis: Analysis of associations between tobacco exposure and triple negative presentation and progesterone expression did not yield any trends or statistically significant correlations (data not shown)

Staging Analysis: A analysis of the association between tobacco exposure and stage 1 - 4 did not show any statistical significant correlations (Table 2).

\section{Discussion}

Tobacco still remains the leading cause of preventable death in the US [1] and causes many illnesses, most notably, cancer. Tobacco has been linked to many cancer types and a causative relationship has been established for some malignancies, such as lung cancer [1]. Carcinogens found in tobacco smoke pass through the alveolar membrane [4] and into the blood stream where they are transported to the breast via plasma lipoproteins [5] [6]. Due to the fact that these carcinogens are lipophilic they can be stored in breast adipose tissue and then metabolized and activated by human mammary epithelial cells [7] [8]. Animal experiments and in vitro studies have shown that compounds found in tobacco smoke, such as polycyclic hydrocarbons, aromatic amines, and N-nitrosamines, may induce mammary tumors [9]. The findings of smoking specific DNA adducts and p53 gene mutations in the breast tissue of smokers [2] further support the biological plausibility of a positive association between cigarette smoking and breast cancer, as does the detection of carcinogenic activity in breast fluid [2].

However epidemiological studies are present mixed results regarding the effect of tobacco in breast cancer risk. Most studies point towards slightly increased risk, but others null or even inverse association [2] [3]. The heterogeneous results may be in part due to the fact that breast cancer is a diverse group of diseases that are caused by different pathological mechanisms [2]. Two thirds of breast cancers are ER and or PR positive tumors which are caused by hormones [3]. Estrogen is a well known risk for breast cancer and this has lead researchers to study ER and PR status in tobacco users yielding mixed results [2] [3]. Furthermore, recent reviews suggest that breast cancer risk differs in pre menopausal vs. post menopausal women [3].

Roughly two thirds to three quarters of breast cancers are ER/PR dependent while the other third to one fifth of breast cancers are associated with over-expression of Human Epidermal growth factor Receptor 2 (HER-2). His receptor is notable for its role in the pathogenesis of breast cancer and as a target for treatment. It is a cell membrane surface-bound receptor tyrosine kinase and is normally involved in the signal transduction pathways leading to cell growth and differentiation.

\begin{tabular}{|c|c|c|c|c|}
\hline & & \multicolumn{2}{|c|}{ Smoke Exposure } & \multirow{2}{*}{ Total } \\
\hline & & No & Yes & \\
\hline \multirow{2}{*}{ I } & Count & 194 & 63 & 257 \\
\hline & \% within Smoke Exposure & $23.4 \%$ & $23.7 \%$ & $23.5 \%$ \\
\hline \multirow{2}{*}{ II } & Count & 285 & 87 & 372 \\
\hline & \% within Smoke Exposure & $34.4 \%$ & $32.7 \%$ & $34.0 \%$ \\
\hline \multirow{2}{*}{ III } & Count & 269 & 86 & 355 \\
\hline & \% within Smoke Exposure & $32.5 \%$ & $32.3 \%$ & $32.4 \%$ \\
\hline \multirow{2}{*}{ IV } & Count & 80 & 30 & 110 \\
\hline & \% within Smoke Exposure & $9.7 \%$ & $11.3 \%$ & $10.1 \%$ \\
\hline \multirow{2}{*}{ Total } & Count & 828 & 266 & 1094 \\
\hline & \% within Smoke Exposure & $100.0 \%$ & $100.0 \%$ & $100.0 \%$ \\
\hline
\end{tabular}


Approximately 15\% - 20\% percent of breast cancers have an amplification of the HER2 gene or overexpression of its protein product. Overexpression of this receptor in breast cancer is associated with increased disease recurrence and worse prognosis. Because of its prognostic role as well as its ability to predict response to trastuzumab, breast tumors are routinely checked for overexpression of HER2. Overexpression also occurs in other cancer such as ovarian cancer, stomach cancer, and biologically aggressive forms of uterine cancer, such as uterine serous endometrial carcinoma. These cancers have a proven association with tobacco consumption. HER2 breast cancer has some estrogen dependent pathways, but not as much as HER2 negative breast cancers with ER and PR positive markers. Therefore it can be postulated that tobacco consumption may lead to HER2 overexpressing breast cancer more so than HER2 negative breast cancers. Currently there is no evidence on the relationship between risk of HER2 breast cancer and tobacco use. We found non-statistically significant positive associations in all analyses between Her-2 expression with or without ER expression and tobacco exposure when analyzed by ethnicity. This is suggestive that there is a potential positive association between tobacco use and HER2 positive breast cancer. A larger number of patients should be investigated in order to clarify this relationship.

\section{Conflict of Interest Statement}

All authors have nothing to disclose.

\section{References}

[1] Fiore, M.C., et al. (2000) Treating Tobacco Use and Dependence. US Department of Health and Human Services, Washington DC.

[2] Terry, P.D. and Rohan, T.E. (2002) Cigarette Smoking and the Risk of Breast Cancer in Women: A Review of the Literature. Cancer Epidemiology, Biomarkers \& Prevention, 11, 953-971.

[3] Band, P.R., et al. (2002) Carcinogenic and Endocrine Disrupting Effects and Risk of Breast Cancer. The Lancet, 260, 1044-1049. http://dx.doi.org/10.1016/S0140-6736(02)11140-8

[4] Yamasaki, E. and Ames, N.B. (1977) Concentration of Mutagens From Urine by Absorption with the Nonpolar Resin XAD-2: Cigarette Smokers Have Mutagenic Urine. Proceedings of the National Academy of Sciences of the United States of America, 74, 3555-3559. http://dx.doi.org/10.1073/pnas.74.8.3555

[5] Plant, A.L., Benson, D.M., Smith, L.C. (1985) Cellular Uptake and Intracellular Localization of Benzo(a) Pyrene by Digital Fluorescence Imaging Microscopy. The Journal of Cell Biology, 100, 1295-1308. http://dx.doi.org/10.1083/jcb.100.4.1295

[6] Shu, H.P. and Bymun, E.N. (1983) Systemic Excretion of Benzo(a) Pyrene in the Control and Microsomally Induced Rat: The Influence of Plasma Lipoproteins and Albumin as Carrier Molecules. Cancer Research, 43, 485-490.

[7] Hech, S.S. (2002) Tobacco Smoke Carcinogens and Breast Cancer. Environmental and Molecular Mutagenesis, 39, 119-126. http://dx.doi.org/10.1002/em.10071

[8] Morris, J.J. and Seifter, E. (1992) The Role of Aromatic Hydrocarbons in the Genesis of Breast Cancer. Medical Hypotheses, 38, 177-184. http://dx.doi.org/10.1016/0306-9877(92)90090-Y

[9] Obana, H., Hori, S., Kashimoto, T. and Kunita, N. (1981) Polycyclic Aromatic Hydrocarbons in Human Fat and Liver. Bulletin of Environmental Contamination and Toxicology, 27, 23-27. 